\title{
Conformation and Aggregation of LK $\alpha 14$ Peptide in Bulk Water and at the Air/Water Interface
}

\author{
Supporting Information
}

\author{
Cahit Dalgicdir ${ }^{\dagger, \ddagger}$ and Mehmet Sayar, ${ }^{*,+}$, \\ College of Engineering, Koç University, Istanbul, Turkey \\ E-mail: msayar@ku.edu.tr
}

Phone: +90 (0)212 3381840. Fax: +90 (0)212 3381548

${ }^{*}$ To whom correspondence should be addressed

${ }^{\dagger}$ College of Engineering, Koç University, Istanbul, Turkey

${ }^{\ddagger}$ Current address: Theoretical Chemistry, University of Konstanz, Konstanz, Germany

"Chemical \& Biological Engineering and Mechanical Engineering Departments, Koç University, Istanbul, Turkey 


\section{Analysis of the REMD Results}

Even though REMD is a very powerful method for sampling the phase space, when the molecule displays rather drastic conformational changes, the convergence of the results is a major challenge. In this study we used REMD for investigating the bulk and interface behavior of a single LK $\alpha 14$ peptide. At the interface as all of the replicas, regardless of the temperature, converge to the $\alpha$-helix conformation the convergence was not a major problem. However in bulk, with the LK $\alpha 14$ peptide displaying several different conformations, a careful analysis of the convergence was required.

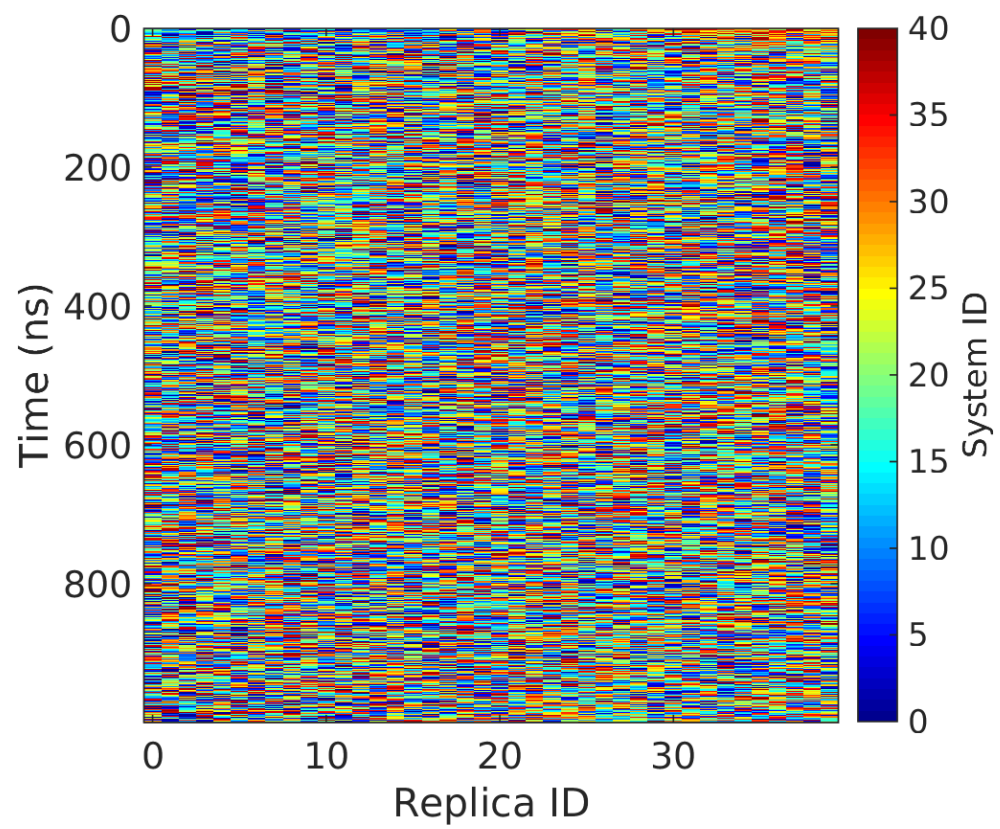

Figure S1: System swaps between neighboring replicas (with different temperatures) through the one microsecond REMD simulation. The horizontal axis represents the replica ids and the color bar represents the system ids.

In Fig. S1 system swaps between neighboring replicas through the one microsecond REMD simulation in bulk water is shown. The mixing of the colors and the average exchange probability of 0.19 demonstrates the quality of the REMD simulation. The snapshot from all the replicas (Fig. S2) and the associated movie display the variety and dynamism of the conformational space of the LK $\alpha 14$ peptide.

In order to check the reliability of the conformational sampling we have done two separate 




Figure S2: Movie depicting the conformational transitions observed in all forty systems through the course of the one microsecond REMD simulation.

analysis one at the residue and the other at the molecular level. At the residue level by using DSSP algorithm for any given frame a secondary structure is assigned to all the residues. The trajectory is divided into four blocks of $250 \mathrm{~ns}$ each. The probability distribution for the assigned secondary structures are calculated for the $\alpha$-helix, $\beta$-sheet or random-coil structures. In Fig. S3 the comparison of the probability distributions in the four blocks are shown. Except for the first block, which covers the first $250 \mathrm{~ns}$ of the $1 \mu$ s simulation, the probability distribution of the DSSP secondary structure assignment does not change.

Next, we performed an analysis for the convergence considering the secondary structure of the whole molecule. By using the complete $1 \mu$ s simulation a merged trajectory of 20000 frames, which includes 500 frames from each replica, is created and clustering analysis is performed. The analysis yields 564 clusters in total, out of which the first eight most populated clusters cover roughly $52 \%$ of the frames. The representative snapshots from these eight clusters and their frequencies are given in Fig. S4.

Now we can analyze the frequency of occurrence of a given cluster at different temper- 




b)


Figure S3: Block analysis of probability of finding a residue in $\alpha$-helix (top), $\beta$-sheet (middle) or random coil (bottom). 
a)

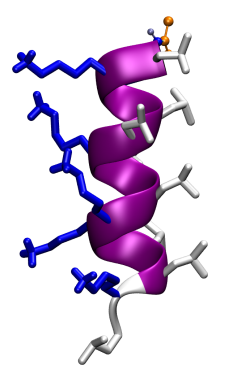

1

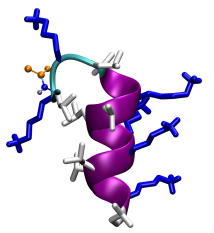

5



2

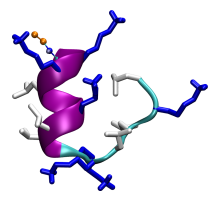

6



3

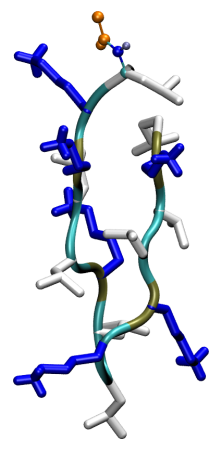

7



4

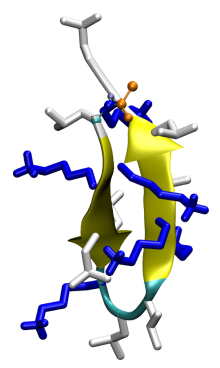

8

b)

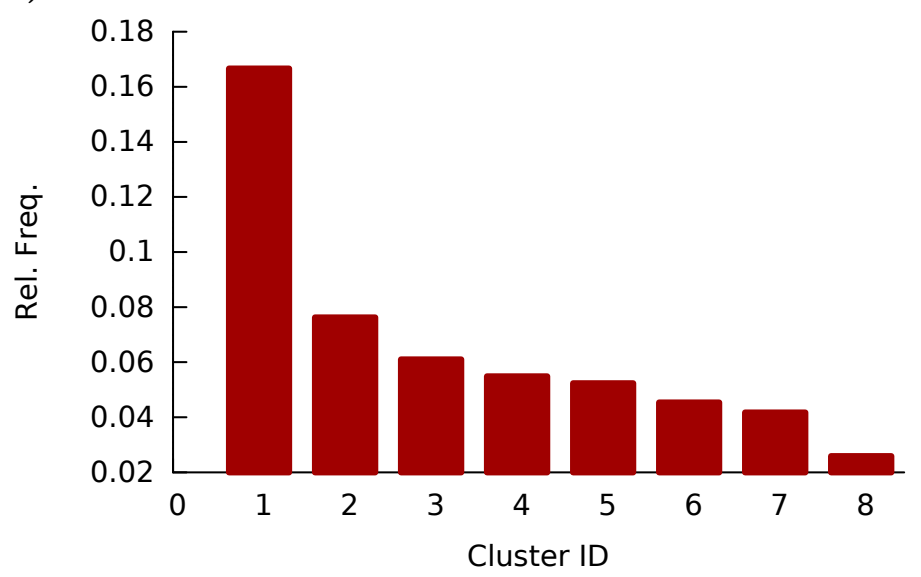

Figure S4: The representative conformations and the relative frequency distribution of the top eight most populated clusters. The clustering analysis was based on a merged trajectory of 20000 frames in total which includes 500 frames from each replica. 
atures and in different time blocks (250 ns blocks of the whole simulation). The frequency distribution as a function of temperature is shown in Fig. S5. Similar to the residue based analysis, here also one can see that the first 250 ns. yields relatively different results compared to the remaining three blocks. Hence, for the cluster analysis of the conformations of the whole molecule at $298 \mathrm{~K}$, as presented in the main text, we have discarded the first 250 ns.



a) cluster 1

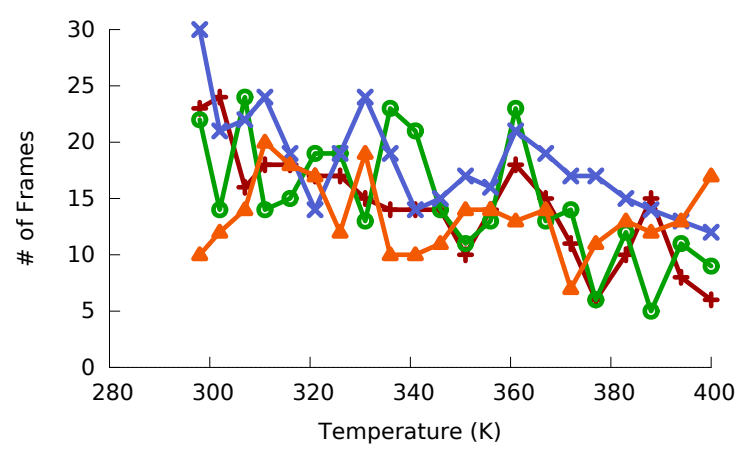

c) cluster 3

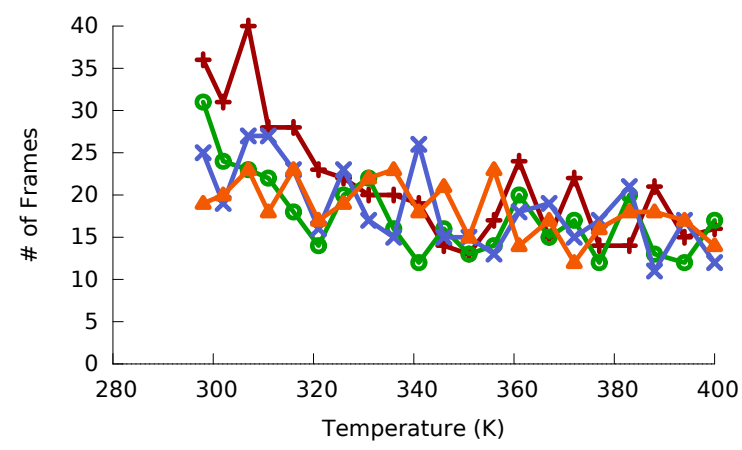

b) cluster 2



d) cluster 4

Figure S5: Convergence check for the top four most populated clusters by block analysis. The number of observed frames for each cluster is given as a function of temperature. The first cluster, which is an $\alpha$-helix, is converged after $250 \mathrm{~ns}$. Hence, the first $250 \mathrm{~ns}$ is treated as equilibration and discarded from the following analysis. In all plots the same line types are used for the time blocks and labels are shown only in part (a). 


\section{I.I REMD analysis for $298 \mathrm{~K}$}

As discussed above the first $250 \mathrm{~ns}$. of the $1 \mu \mathrm{s}$ simulation was considered as equilibration of the REMD simulation and all of the analysis was made over the remaining $750 \mathrm{~ns} .37500$ frames were chosen from the complete REMD trajectory at $298 \mathrm{~K}$. For this trajectory clustering analysis yields 294 clusters in total, where the frequency distribution is given in Fig. S6. The details of the clustering algorithm are given in Methods section of the manuscript. As the frequency distribution does not yield a clear cut-off, we have decided to focus on the first eight clusters, which include roughly half of all the frames used in the cluster analysis.

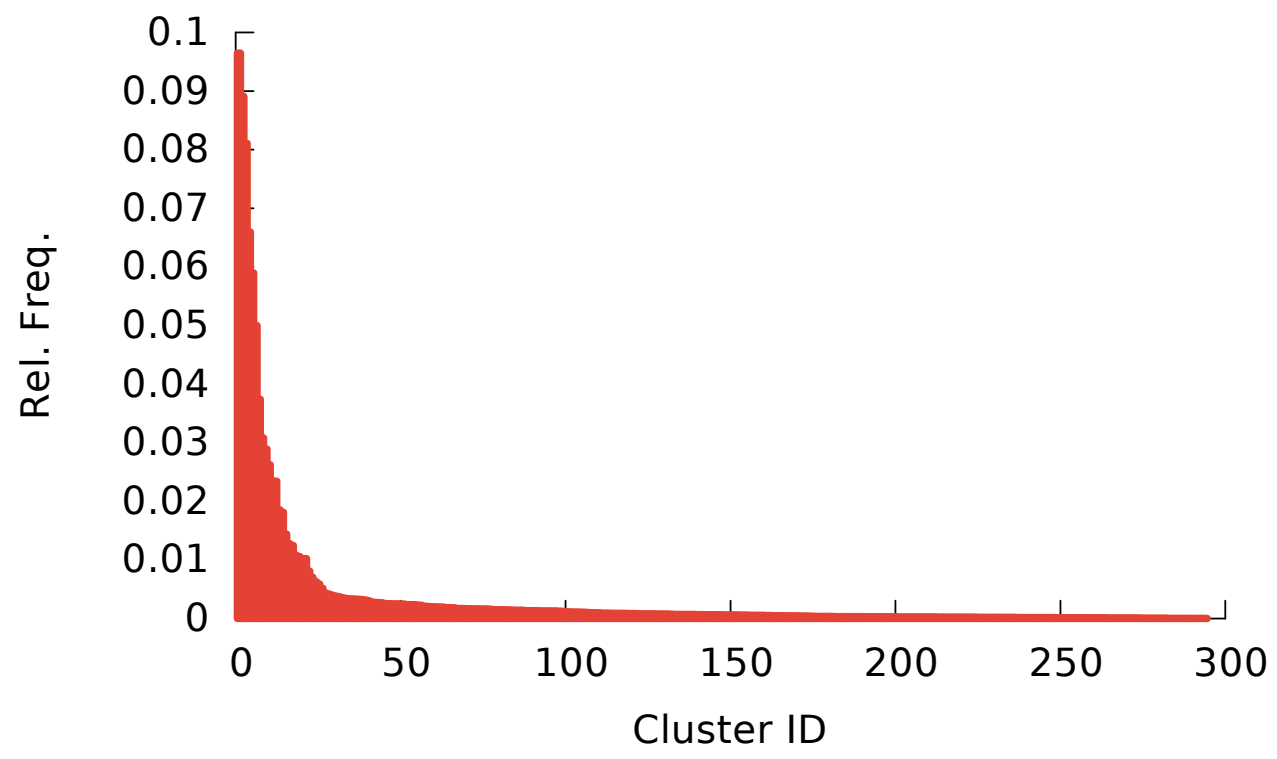

Figure S6: Relative frequency of occurrence of the clusters as obtained from hierarchical clustering.

For these eight clusters the data is divided into three blocks of $250 \mathrm{~ns}$ each to compare the frequencies of the clusters in different time blocks. Based on the distributions shown in Fig. S7 we have concluded that the $1 \mu$ s simulation is converged and adequate conformational sampling of LK $\alpha 14$ in bulk water is achieved. 


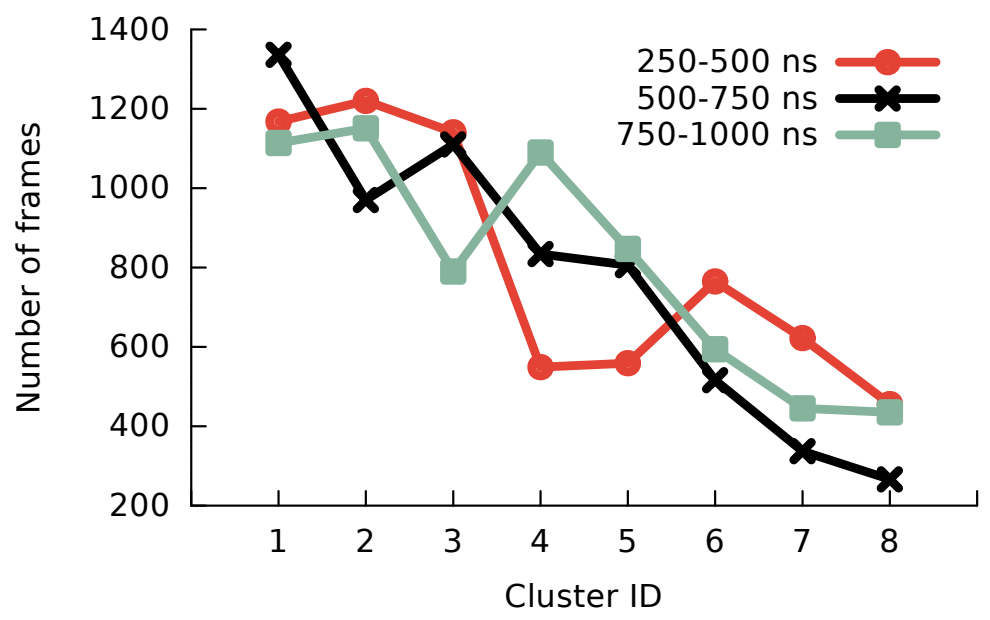

Figure S7: Block analysis for the first 8 clusters for the replica at $298 \mathrm{~K}$. 


\section{PMF analysis for adsorption to the interface}

For calculating the adsorption free energy of the LK $\alpha 14$ molecule to the vacuum/water interface umbrella sampling simulations were performed. In order to decompose the adsorption free energy we have performed simulations with the molecule in helix and random coil conformations. The umbrella sampling simulations were run for $200 \mathrm{~ns}$ for each window. To check the convergence of the results block analysis is performed. The first 50 ns of the simulation is discarded for equilibration. The analysis results given in Fig. $\mathrm{S} 8$ is based on the remaining trajectory.

a)

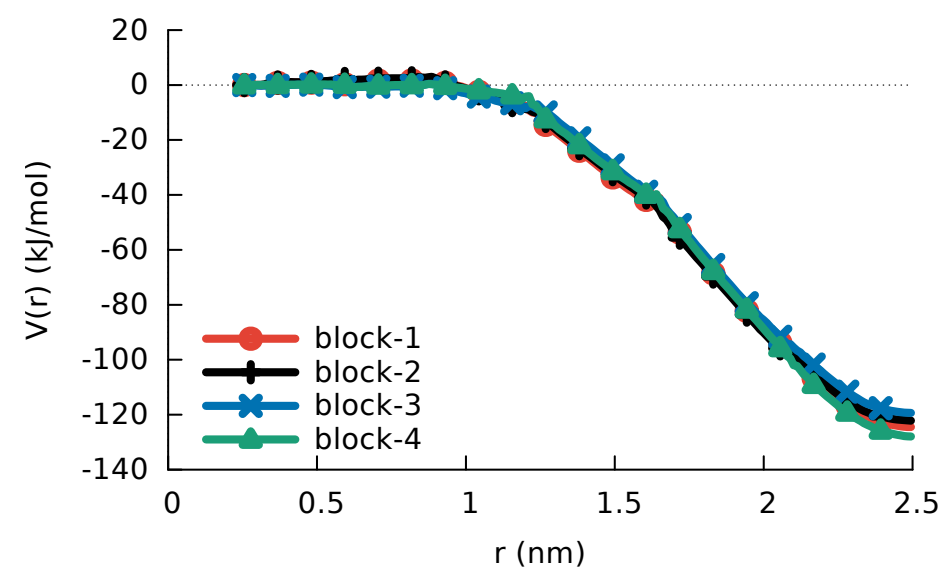

b)



Figure S8: Block analysis for the umbrella sampling simulation of a single helical LK $\alpha 14$ at the vacuum/water interface for helical (top) and coil (bottom) peptide. 
Since LK $\alpha 14$ peptide is free to change its conformation during the simulations these umbrella sampling simulations, especially in the random coil conformation, does not represent true thermodynamic equilibrium. The equilibrium conformation of the peptide at the interface is $\alpha$-helix, therefore the random-coil conformation should eventually transform into helix given sufficient time. The DSSP analysis of the umbrella sampling windows is given in Fig. S9. The DSSP plots are arranged such that the distance to the interface is decreasing from left to right and top to bottom. For windows where the molecule is at the interface the $\alpha$-helix conformation is preserved. However, for intermediate distances where the molecule cannot fully dehydrate its leucine side chains, partial unfolding is seen. In this manner the molecule can position a portion of its leucine side chains at the interface while it is under the influence of the restraining force from umbrella sampling setup. The random coil conformation is clearly not truly random either. It folds into a partial $\beta$-hairpin structure in some of the umbrella windows (Fig. S9 bottom). However, as the conformation is still far away from being an $\alpha$-helix, the PMF curve can still be used to compare the energy difference between helix and random coil conformations. 
a)
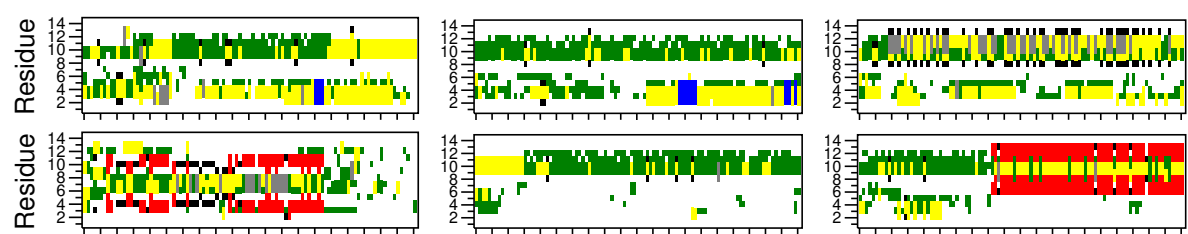


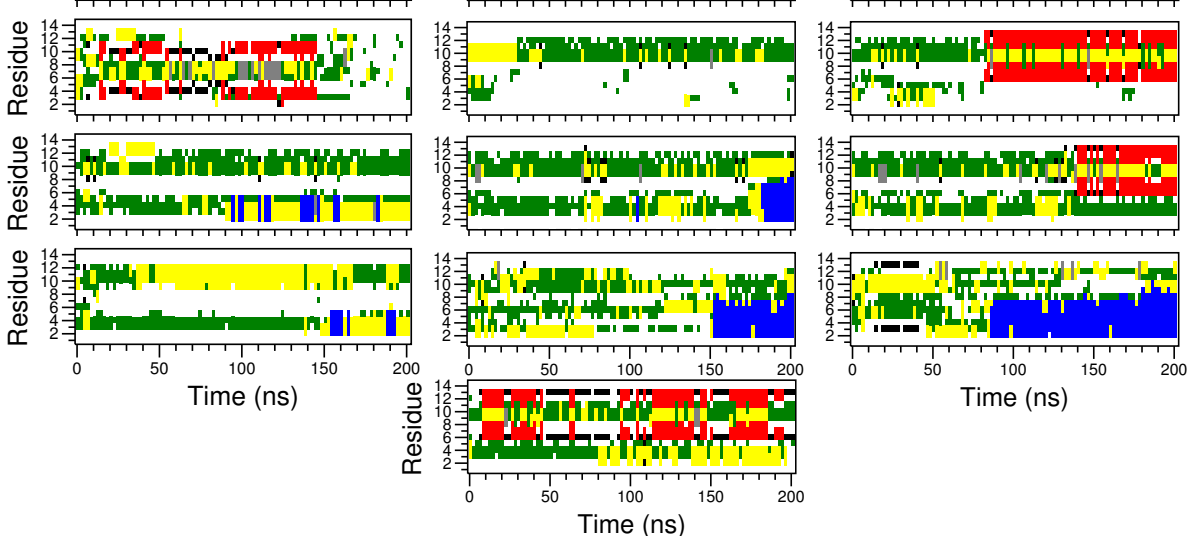

b)
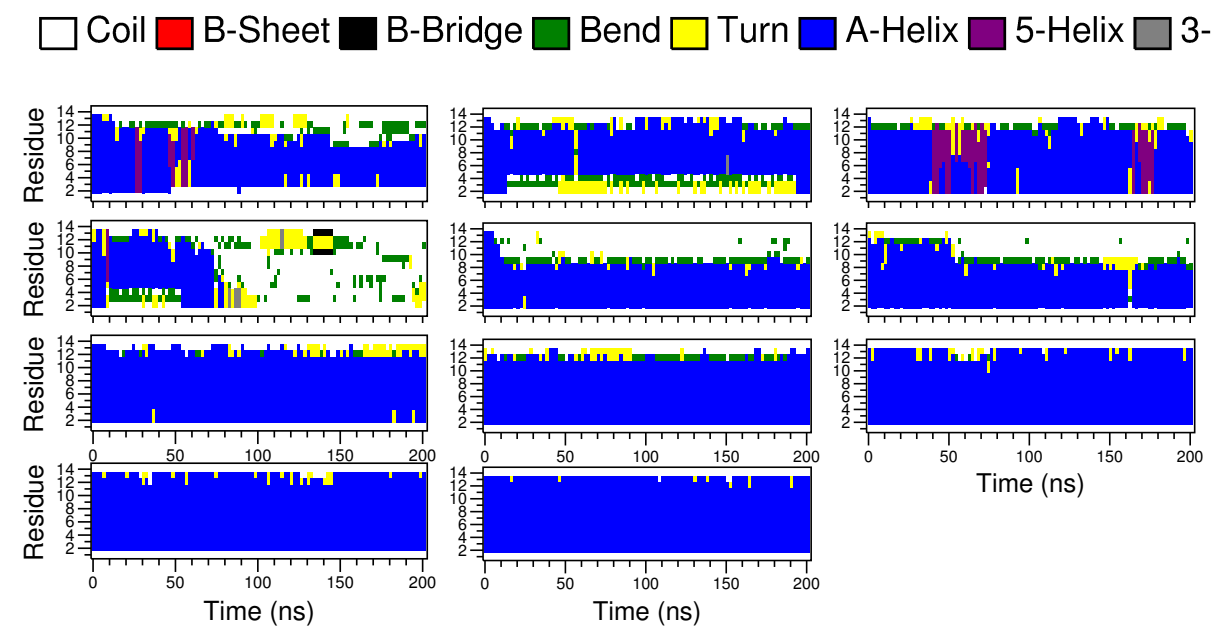

Figure S9: DSSP plots for umbrella simulations at the vacuum/water interface with $\alpha$-helix (top) and random coil LK $\alpha 14$ peptides. Separate windows correspond to separate distances to the interface, where the distance decreases from left to right and top to bottom. 


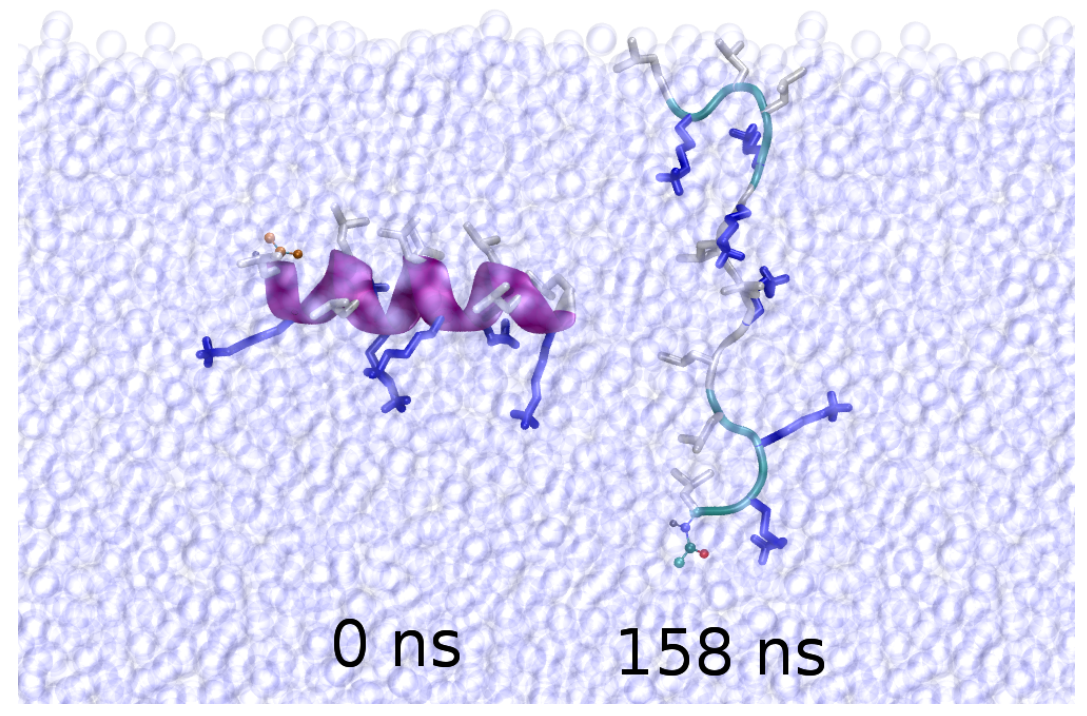

Figure S10: Snapshots from the fourth window of umbrella sampling, which corresponds to a $1.5 \mathrm{~nm}$ depth from the interface. Initially $\alpha$-helical peptide unfolds to make contact with the interface. 


\section{Aggregation}

The correlation plot in Fig. 7 were produced from the timeline data in Fig. S11. For the parallel and anti-parallel orientations the distance and angle between the two molecules is given as a function of simulation time. The probability plots are also given for the same data in the bottom left (distance) and right (angle) panels.

All the peptides in the aggregates of size 3,4,5, and 8 remain helical during the course of the simulations as seen from the DSSP analysis in Fig. S12. For the tetramer correlation of inter-peptide distance vs inter-peptide angle for each of the peptide pairs of tetramer in bulk water is shown in Fig. S13.

The data given in the bar chart presented in Fig. 10 is based on the timeline data shown in Fig. S14. 

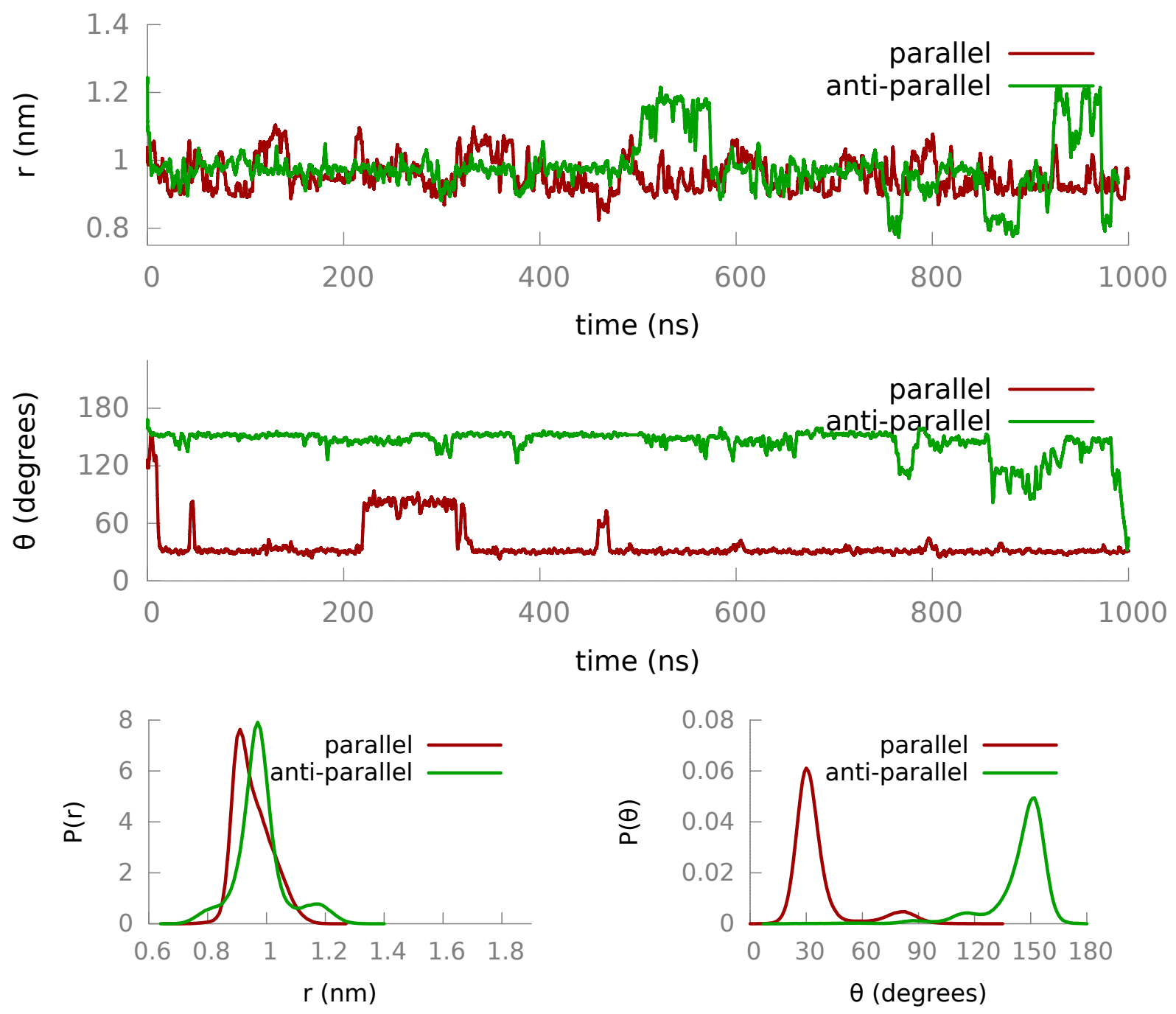

Figure S11: Comparison of parallel and anti-parallel orientation of LK $\alpha 14$ dimer in bulk water: Timeline of inter-dimer distance and inter-dimer angle (top) and the corresponding histograms (bottom). The distance is the distance between the centers of mass distance of the backbone atoms. The angle is the angle between the vectors passing through the backbone of the peptides, where the first and last 3 residues are not taken into account due to occasional unfolding of the end residues. The parallel orientation adopts an average inter-dimer angle of $30^{\circ}$ and an inter-dimer distance of $0.97 \mathrm{~nm}$ where the anti-parallel one yields $150^{\circ}$ and $1.06 \mathrm{~nm}$. 


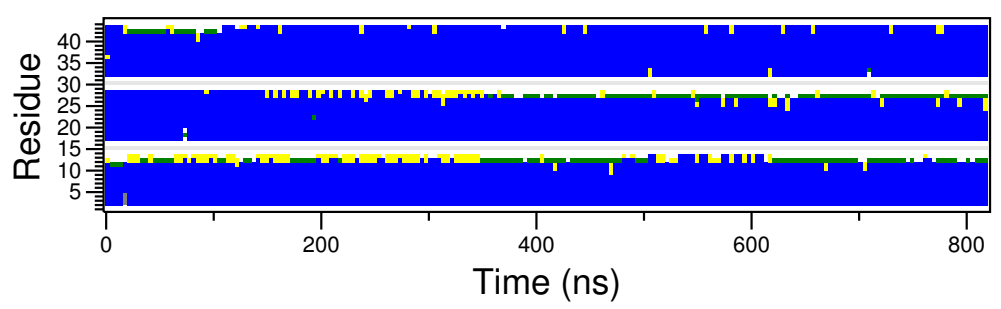

(a) trimer



(b) tetramer

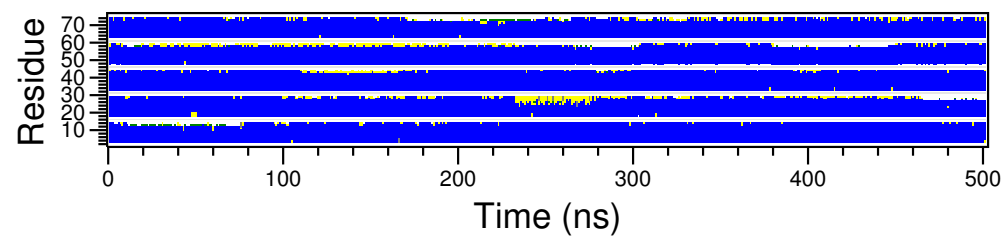

(c) pentamer



$\square$ Coil $\square$ Bend $\square$ Turn $\square$ A-Helix $\square$ 5-Helix $\square$ 3-Helix $\square$ Chain_Separatc

(d) octamer

Figure S12: DSSP analysis of the time evolution of the secondary structure for each residue for trimer, tetramer, pentamer and octamer in bulk water color coded for each secondary structure. 



Figure S13: Correlation of inter-peptide distance vs inter-peptide angle for each of the peptide pairs of tetramer in bulk water. 

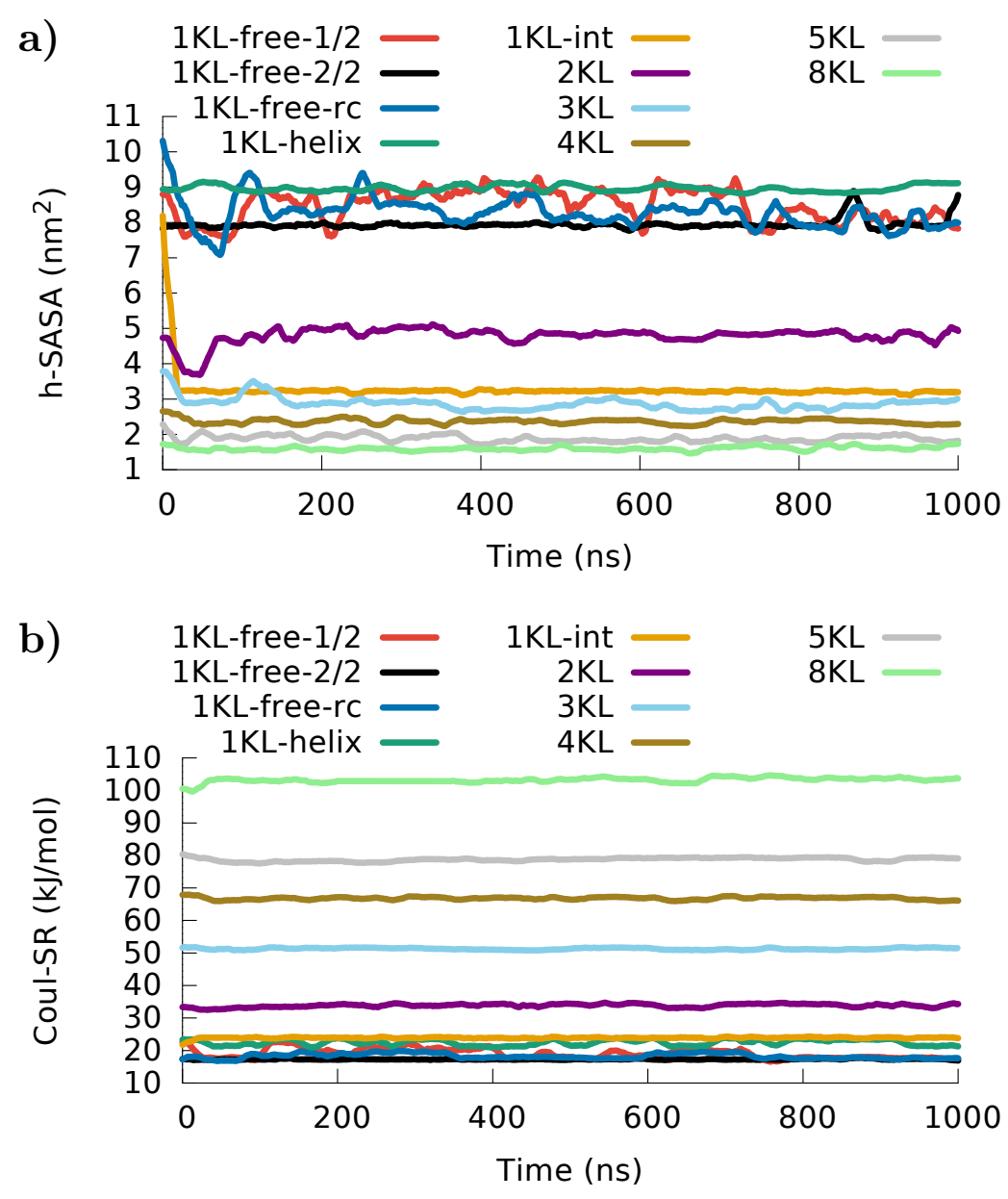

Figure S14: The interplay between hydrophobic forces acting on the leucine side chains (a) and Coulomb repulsion (b) determine the aggregate stability. 


\section{Aggregation at the interface}

As discussed in the main text the conformational space of $L K \alpha 14$ peptide is reduced to the $\alpha$-helix at the vacuum/water interface. This observation at the single molecule level is also confirmed in our simulations for the aggregation behavior of LK $\alpha 14$ at the interface. The DSSP analysis for the two peptides is shown in Fig. S15.

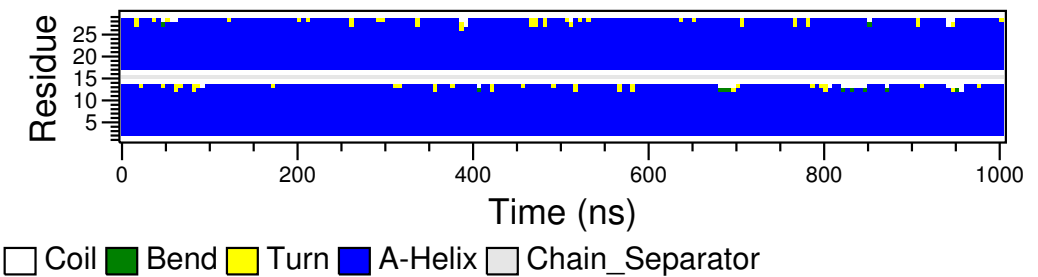

Figure S15: Time evolution of the secondary structure for a dimer at the vacuum/water interface.

Initial configuration for the simulation with eight LK $\alpha 14$ peptides at the interface is given in Fig. S16. Note that only the portion of the box which includes the molecules are given. The molecules are aligned in anti-parallel orientation in close contact near the vacuum/water interface. The dimensions of the box were $14 \mathrm{~nm}, 14 \mathrm{~nm}, 18 \mathrm{~nm}$ along $\mathrm{x}, \mathrm{y}$, and $\mathrm{z}$ dimensions, respectively, containing 38272 water molecules and the water slab is $6 \mathrm{~nm}$ long.
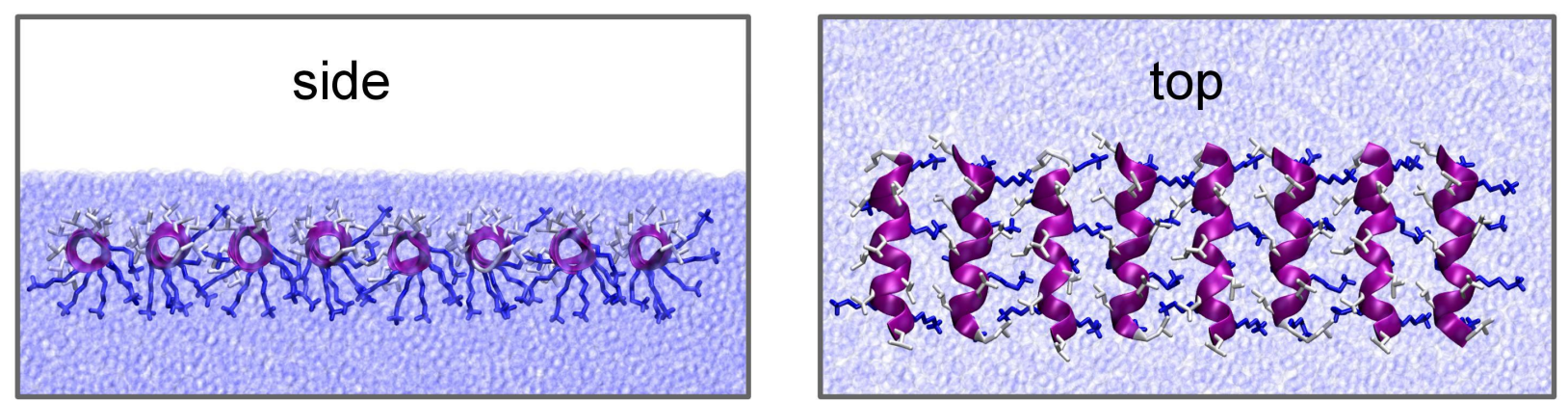

Figure S16: The initial configuration for eight LK $\alpha 14$ peptides at the vacuum/water interface. Note that the actual box size is larger than the depicted area. The system has 38272 water molecules and the box is $14 \mathrm{~nm}, 14 \mathrm{~nm}, 18 \mathrm{~nm}$ long along $\mathrm{x}, \mathrm{y}$ and $\mathrm{z}$ dimensions, respectively, with a $6 \mathrm{~nm}$ long water slab.

During the course of the simulation all eight peptides remain in $\alpha$-helix form as demonstrated by the DSSP analysis in Fig. S17. 


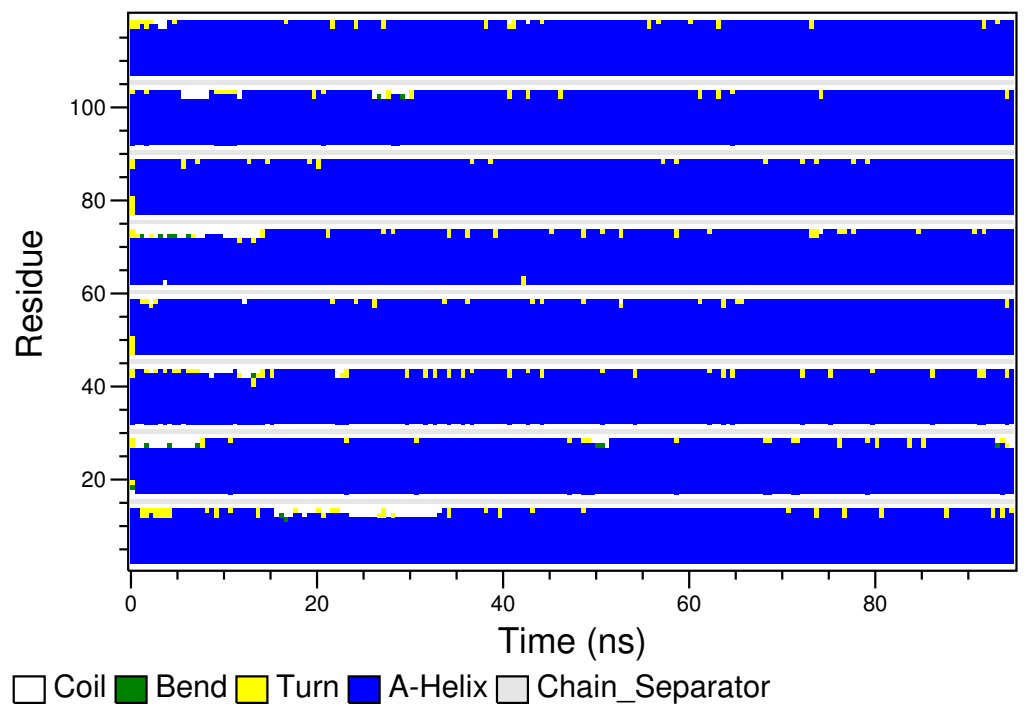

Figure S17: Time evolution of the secondary structure for eight LK $\alpha 14$ peptides simulated at the vacuum/water interface. Except intermittent unfolding of the end residues, $\alpha$-helix structure is stable for all peptides.

To check the convergence of the PMF curves presented in Fig. 12 block analysis is performed (Fig. S18). Note that since the orientation is completely lost beyond $2 \mathrm{~nm}$, the same windows are used in calculation of the PMF curves for anti-parallel and parallel. 
a)

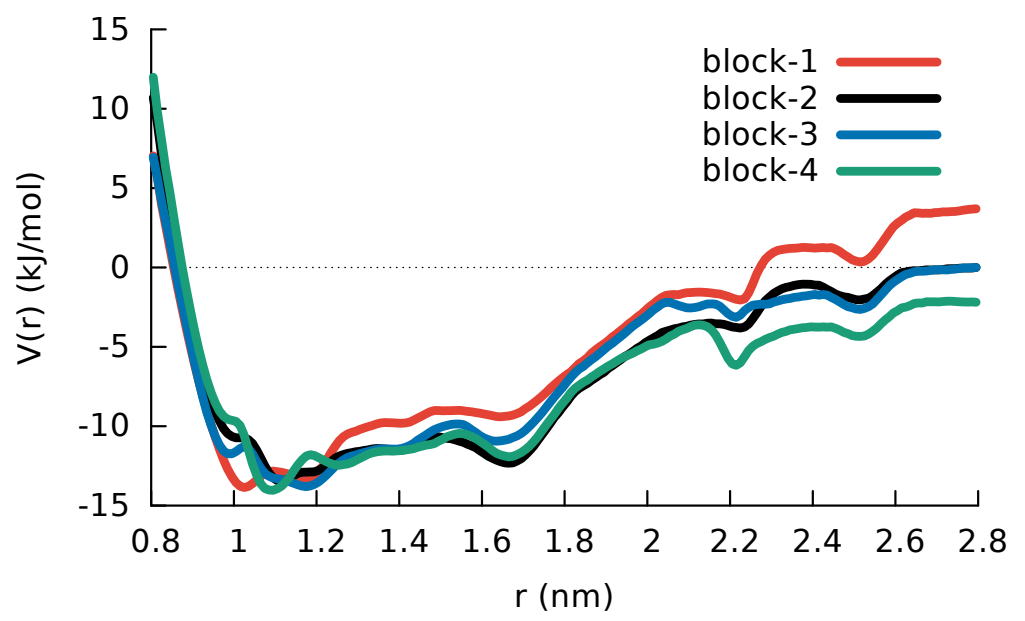

b)



Figure S18: Block analysis for the umbrella sampling simulations for the dimers at the vacuum/water interface, for the anti-parallel (top) and the parallel (bottom) cases. Each windows was run for $500 \mathrm{~ns}$ and $350 \mathrm{~ns}$ for the anti-parallel and parallel cases, respectively. The initial $50 \mathrm{~ns}$ were regarded as equilibration and therefore discarded. 